\title{
The Random Component of Mixer-Based Nonlinear Vector Network Analyzer Measurement Uncertainty
}

Peter Stuart Blockley, Member, IEEE, Jonathan Brereton Scott, Senior Member, IEEE, Daniel Gunyan, Senior Member, IEEE, and Anthony Edward Parker, Senior Member, IEEE

\begin{abstract}
The uncertainty, due to random noise, of the measurements made with a mixer-based nonlinear vector network analyzer are analyzed. An approximate covariance matrix corresponding to the measurements is derived that can be used for fitting models and maximizing the dynamic range in the measurement setup. The validity of the approximation is verified with measurements.
\end{abstract}

Index Terms-Nonlinear systems, phase measurement, uncertainty.

\section{INTRODUCTION}

A MIXER-BASED measurement system, commonly referred to as a nonlinear vector network analyzer (NVNA) or large signal network analyzer (LSNA), ${ }^{1}$ which performs wide-bandwidth measurements of signals and components, has been reported [1]. The system measures complex modulated signals at two ports that are calibrated to coaxial or on-wafer measurement planes.

The measurement system not only returns the magnitude, but also the phase of the signal spectrum being measured, maintaining phase relationships between frequency components relative to a reference tone. The phase information is useful for analyzing distortion or wide-bandwidth signal analysis. With wide-bandwidth magnitude and phase information, the measurements can be transformed to the time domain such that the crest factor can be calculated or signal clipping observed.

The system has applications in signal analysis, nonlinear component tests, time-domain measurements, measurement of frequency translating devices, device linearization, and device modeling. For many of these applications, an understanding of measurement uncertainty is important for maximizing dynamic range in the measurement setup, determining phase using alignment algorithms, developing models from measurement data, and calculating uncertainty bounds.

The measurements from the system have uncertainty due to random noise and systematic errors. The instrument calibration reduces systematic errors due to linear time-invariant systematic processes. However, the uncertainty due to other system-

Manuscript received March 17, 2007.

P. S. Blockley was with the Department of Electronics, Macquarie University, Sydney, N.S.W. 2109, Australia. He is now with South Pacific IT, Sydney, N.S.W. 2009, Australia (e-mail: peterblockley@ieee.org).

J. B. Scott is with the School of Science and Engineering, University of Waikato, Hamilton, New Zealand (e-mail: jonathanscott@ieee.org).

D. Gunyan is with the Microwave Technology Center, Agilent Technologies, Santa Rosa, CA 95404 USA (e-mail: daniel.gunyan@ieee.org).

A. E. Parker is with the Department of Electronics, Macquarie University, Sydney, N.S.W. 2109, Australia (e-mail: tonyp@ ieee.org)

Digital Object Identifier 10.1109/TMTT.2007.906515

${ }^{1}$ This paper uses the acronym NVNA, although LSNA is also commonly used.

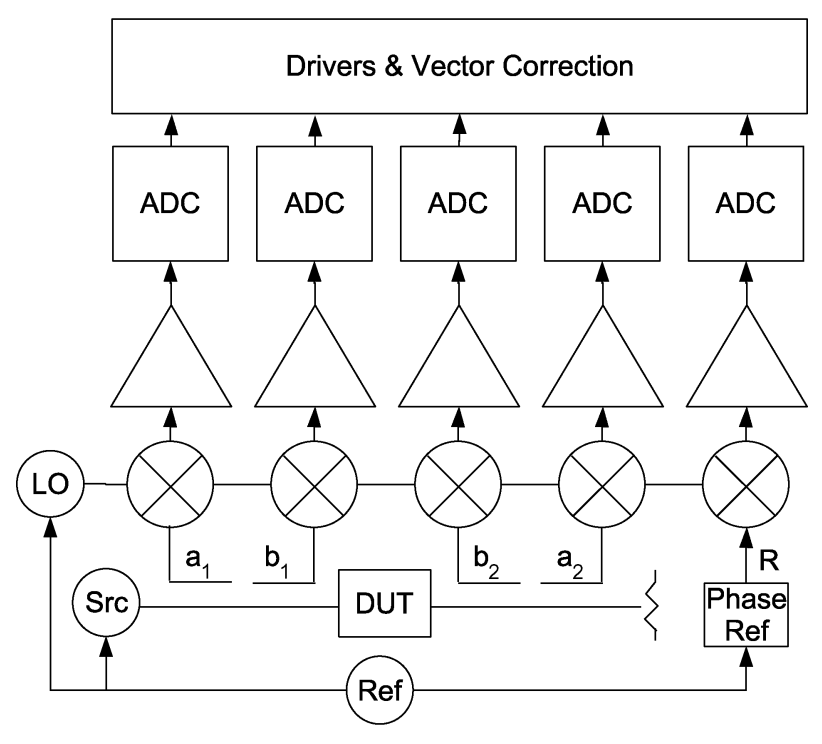

Fig. 1. Block diagram of the measurement system. The hardware consists of five receivers and four directional networks. The receivers measure the incident and reflected waves via the directional networks connected to each port. The fifth receiver measures a phase reference (Phase Ref). The measurement system, source (Src), and phase reference are phase locked to a common reference (Ref).

atic distortion and random noise remains. This paper attempts to quantify the random noise by deriving approximate covariance matrices for the system measurements.

The measurement system uses a phase reference to recover the phase from measurements of wide-bandwidth signals. This results in measurement uncertainty, which depends on the receiver noise, the amplitude of the signal, amplitude of the phase reference, and phase reference noise. In addition, using a phase reference to recover the phase increases the correlation between the measurements.

Section II describes the measurement system architecture. Section III analyzes the uncertainty of the measurements and an approximation for the covariance matrix is derived. Section IV describes an empirically derived covariance matrix that accounts for phase reference noise. Section V presents measurements using the measurement system and compares the variance of the measurements to that predicted by the approximate convariance matrix.

\section{MeAsurement System Architecture}

The measurement system [1] is based on a four-port 20-GHz linear vector network analyzer (Agilent N5230A, option 245) that has been configured for two-port nonlinear operation. The block diagram for the system is shown in Fig. 1. The hardware consists of five receivers and four directional networks. The re- 
ceivers measure the incident and reflected waves via the directional networks connected to each port. The fifth receiver measures a phase reference.

The measurement system operates in the frequency domain in a similar fashion to a multireceiver spectrum analyzer. A local oscillator is swept across a frequency range and the tones of interest are downconverted to a narrow bandwidth IF where they are sampled. The local oscillator synthesizer is not phase coherent with the tones of interest, and the phase is unknown. Additionally, changing the local oscillator frequency results in a new local oscillator phase. Therefore, the phase information is only valid in the bandwidth of the IF filter for a single local oscillator frequency.

The measurement system recovers the phases of the tones of interest by using a single shared local oscillator applied to each receiver and, at the same time, measuring a signal with known relative phase between its tones. In practice, the known signal (generated by a phase reference) is an impulse train in the time domain that is highly repeatable and can be made traceable to the electrooptic sampling system of the National Institute of Standards and Technology (NIST), Boulder, CO [2].

The system acquires signals from the five receivers, simultaneously measuring the forward pseudowave ${ }^{2}$ at Port $1, a_{1}^{m}$, reverse pseudowave at Port $1, b_{1}^{m}$, forward pseudowave at Port 2, $a_{2}^{m}$, reverse pseudowave at Port $2, b_{2}^{m}$, and reference $R^{m}$. Superscript $m$ denotes a measured quantity rather than an actual or transformed quantity. Subscript 1 refers to the pseudowave at Port 1 before calibration and subscript 2 refers to the pseudowave at Port 2 before calibration. The scope of this paper will be limited to the uncertainty, due to random noise, of the pseudowaves before calibration. The phases of the measured waves $\left(a_{1}^{m}, b_{1}^{m}, a_{2}^{m}, b_{2}^{m}\right.$, and $\left.R^{m}\right)$ have a component due to the phase of the actual waves at the receiver $\left(a_{1}^{\prime}, b_{1}^{\prime}, a_{2}^{\prime}, b_{2}^{\prime}\right.$, and $\left.R^{\prime}\right)$ and a component due to the phase of the local oscillator $\left(\phi_{\mathrm{lo}}\right)$. The measured phase at a single frequency can, therefore, be expressed as

$$
\begin{aligned}
& \varphi\left(a_{1}^{m}\right)=\varphi\left(a_{1}^{\prime}\right)+\phi_{\mathrm{lo}} \\
& \varphi\left(b_{1}^{m}\right)=\varphi\left(b_{1}^{\prime}\right)+\phi_{\mathrm{lo}} \\
& \varphi\left(a_{2}^{m}\right)=\varphi\left(a_{2}^{\prime}\right)+\phi_{\mathrm{lo}} \\
& \varphi\left(b_{2}^{m}\right)=\varphi\left(b_{2}^{\prime}\right)+\phi_{\mathrm{lo}} \\
& \varphi\left(R^{m}\right)=\varphi\left(R^{\prime}\right)+\phi_{\mathrm{lo}}
\end{aligned}
$$

where $\varphi(x)$ is a function that returns the argument of $x$ (the angle associated with the complex quantity $x$ ).

The dependence of the measurement quantities on the local oscillator phase $\phi_{\text {lo }}$ can be removed by subtracting the reference receiver phase from that of the measurement quantity. For example, the phase of the transformed forward pseudowave $\varphi\left(a_{1}\right)$ would be given by

$$
\begin{aligned}
\varphi\left(a_{1}\right) & =\varphi\left(a_{1}^{m}\right)-\varphi\left(R^{m}\right) \\
& =\left[\varphi\left(a_{1}^{\prime}\right)+\phi_{\mathrm{lo}}\right]-\left[\varphi\left(R^{\prime}\right)+\phi_{\mathrm{lo}}\right] \\
& =\varphi\left(a_{1}^{\prime}\right)-\varphi\left(R^{\prime}\right) .
\end{aligned}
$$

\footnotetext{
${ }^{2}$ A pseudowave is an wave-like quantity defined in terms of an arbitrary reference impedance [3].
}

The transformed forward pseudowave $a_{1}$ is independent of the local oscillator phase, but perturbed by the phase of the phase reference. Knowledge of the relative phase of the reference can then be used to recover the relative phase of the actual wave.

This operation can also be performed with complex arithmetic. The transformed pseudowaves $\left(a_{1}, b_{1}, a_{2}, b_{2}\right.$, and $\left.R\right)$ corresponding to the raw measurements $\left(a_{1}^{m}, b_{1}^{m}, a_{2}^{m}, b_{2}^{m}\right.$, and $R^{m}$ ) are given by

$$
\begin{aligned}
a_{1} & =\frac{a_{1}^{m}}{R^{m}}\left|R^{m}\right| \\
b_{1} & =\frac{b_{1}^{m}}{R^{m}}\left|R^{m}\right| \\
a_{2} & =\frac{a_{2}^{m}}{R^{m}}\left|R^{m}\right| \\
b_{2} & =\frac{b_{2}^{m}}{R^{m}}\left|R^{m}\right| \\
R & =\frac{R^{m}}{R^{m}}\left|R^{m}\right|
\end{aligned}
$$

where $\left|R^{m}\right|$ is the magnitude of $R^{m}$.

Although the transformed reference signal $R$ has no phase information, it is useful for evaluating the uncertainty of the measurements.

The complex measurement quantities can be expressed in terms of their real and imaginary parts. The real part $\Re\left(a_{1}\right)$ and imaginary part $\Im\left(a_{1}\right)$ of $a_{1}$ are given by

$$
\begin{aligned}
& \Re\left(a_{1}\right)=\frac{\Re\left(a_{1}^{m}\right) \Re\left(R^{m}\right)+\Im\left(a_{1}^{m}\right) \Im\left(R^{m}\right)}{\left|R^{m}\right|} \\
& \Im\left(a_{1}\right)=\frac{\Im\left(a_{1}^{m}\right) \Re\left(R^{m}\right)-\Re\left(a_{1}^{m}\right) \Im\left(R^{m}\right)}{\left|R^{m}\right|}
\end{aligned}
$$

where $\Re\left(a_{1}^{m}\right)$ is the real part of the measured wave $a_{1}^{m}, \Im\left(a_{1}^{m}\right)$ is the imaginary part of the measured wave $a_{1}^{m}, \Re\left(R^{m}\right)$ is the real part of the measured reference receiver $R^{m}$, and $\Im\left(R^{m}\right)$ is the imaginary part of the measured reference receiver $R^{m}$.

The ability to recover the phase information is combined with the ability to measure the forward and reverse waves at each port to enable full vector calibration [1].

\section{Derivation of the Covariance MatriX}

For many applications, such as fitting models and aligning signals, it is important to know the covariance matrix corresponding to the measurements. The covariance matrix can be used to weight a cost function when fitting models, used to weight the signal alignment problem [4] or give uncertainty bounds for the measurements.

While the covariance matrix for the raw measurements $\left(a_{1}^{m}\right.$, $b_{1}^{m}, a_{2}^{m}$, and $b_{2}^{m}$ ) may be known, the measurements undergo a nonlinear transformation (3) in order to remove the dependence of the local oscillator. This section derives the variance of the magnitude and phase for the raw measurement $a_{1}^{m}$, the variance of the magnitude and phase for the transformed pseudowave $a_{1}$, the covariance matrix for the transformed pseudowave $a_{1}$, and the covariance matrix for the four phase measurements $\varphi\left(a_{1}\right)$, $\varphi\left(b_{1}\right), \varphi\left(a_{2}\right)$, and $\varphi\left(b_{2}\right)$. 


\section{A. Single Receiver}

Consider the variance of the measurements obtained from one of the five receivers. When the time-domain samples of the signal are corrupted by stationary narrowband additive Gaussian noise, the complex valued measurements $\left(\Re\left(a_{1}^{m}\right)\right.$ and $\left.\Im\left(a_{1}^{m}\right)\right)$ of the pseudowave $\left(a_{1}^{m}\right)$ are independent with equal variance [5]. The population covariance matrix is given by

$$
\Sigma_{a_{1}^{m}}=\left[\begin{array}{cc}
\sigma^{2} & 0 \\
0 & \sigma^{2}
\end{array}\right]
$$

where $\sigma^{2}$ is the variance of $\Re\left(a_{1}^{m}\right)$ and $\Im\left(a_{1}^{m}\right)$.

The measurements from a single receiver have the advantage that the real and imaginary parts of the complex-valued measurement are independent with equal variance so a least squares method can be used to fit models to the measurement data. In addition, the confidence region for the mean of the measurements is a circle of constant radius around the mean in the complex plane, independent of the signal magnitude.

Often data is presented as magnitude and phase, where the magnitude $\left|a_{1}^{m}\right|$ and phase $\varphi\left(a_{1}^{m}\right)$ are given by ${ }^{3}$

$$
\left[\begin{array}{c}
\left|a_{1}^{m}\right| \\
\varphi\left(a_{1}^{m}\right)
\end{array}\right]=\left[\begin{array}{c}
\sqrt{\Re\left(a_{1}^{m}\right)^{2}+\Im\left(a_{1}^{m}\right)^{2}} \\
\tan ^{-1}\left(\frac{\Im\left(a_{1}^{m}\right)}{\Re\left(a_{1}^{m}\right)}\right)
\end{array}\right] .
$$

This is a nonlinear transformation of the complex valued quantity $a_{1}^{m}$ and, as a result, the distributions of the new variables $\left|a_{1}^{m}\right|$ and $\varphi\left(a_{1}^{m}\right)$ are not Gaussian.

The magnitude $\left|a_{1}^{m}\right|$ has a Rice distribution [6], [7] where the variance of the magnitude $\left|a_{1}^{m}\right|$ is given by [5]

$$
\begin{aligned}
\operatorname{var}\left(\left|a_{1}^{m}\right|\right)= & 2 \sigma^{2}+\left|a_{1}^{m}\right|^{2}-\frac{\pi \sigma^{2}}{2} \exp \left(\frac{-\left|a_{1}^{m}\right|^{2}}{2 \sigma^{2}}\right) \\
\times & {\left[\left(1+\frac{\left|a_{1}^{\prime}\right|^{2}}{2 \sigma^{2}}\right) I_{0}\left(\frac{\left|a_{1}^{\prime}\right|^{2}}{4 \sigma^{2}}\right)\right.} \\
& \left.+\frac{\left|a_{1}^{m}\right|^{2}}{4 \sigma^{2}} I_{1}\left(\frac{\left|a_{1}^{m}\right|^{2}}{4 \sigma^{2}}\right)\right]^{2}
\end{aligned}
$$

where $I_{0}(x)$ and $I_{1}(x)$ are the modified Bessel functions of the first kind.

The variance of the phase $\operatorname{var}\left[\varphi\left(a_{1}^{m}\right)\right]$ must be evaluated from a numerical integration involving the probability density function of $\varphi\left(a_{1}^{m}\right)$.

Often it is of interest to have a simple estimate of the variance. Such an estimate can be made by using a linearization technique. Linearization techniques for estimating the covariance matrix have been implemented in nonlinear least squares software such as NL2SOL [8] and ODRPACK [9]. It has been shown that the linearized method used in this paper is as good, and often better, than the other linearization methods and has the advantage that it is more numerically stable [10].

${ }^{3}$ In practice, $\tan ^{-1}(y / x)$ is implemented with a function, e.g., $\operatorname{atan} 2(x, y)$, which returns a phase from one of four quadrants $(-\pi<\phi \leq \pi)$ rather than two quadrants $(-(\pi / 2)<\phi \leq(\pi / 2))$. The function $\operatorname{atan} 2(x, y)$ must satisfy $\cos [\operatorname{atan} 2(x, y)]=x / \sqrt{x^{2}+y^{2}}$ and $\sin [\operatorname{atan} 2(x, y)]=y / \sqrt{x^{2}+y^{2}}$.

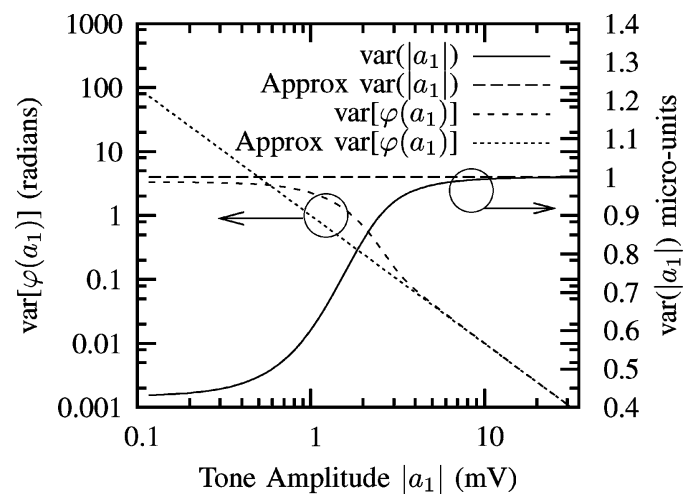

Fig. 2. Graphical example of the approximate variance (11) compared to the variance of the magnitude $\operatorname{var}\left(\left|a_{1}\right|\right)$ and phase $\operatorname{var}\left[\varphi\left(a_{1}\right)\right]$ for different tone amplitudes. The variance of the real and imaginary parts $\left(\Re\left(a_{1}^{m}\right)\right.$ and $\left.\Im\left(a_{1}^{m}\right)\right)$ was $\sigma^{2}=10^{-6}$. The variance of the magnitude was calculated from (8) and the variance of the phase from a numerical integration of the phase probability density function. The approximation is accurate when the tone amplitude $\left|a_{1}\right|$ is much greater than the standard deviation $\sigma$.

Consider the Taylor-series expansion of the function (7) truncated to first order. This approximation results in the following linearized function for the magnitude and phase:

$$
\left[\begin{array}{c}
\left|a_{1}^{m}\right| \\
\varphi\left(a_{1}^{m}\right)
\end{array}\right] \approx \mathrm{A}\left[\Re\left(a_{1}^{m}\right) \quad \Im\left(a_{1}^{m}\right)\right]^{T}
$$

where $A$ is the matrix

$$
\mathrm{A}=\left[\begin{array}{cc}
\frac{\Re\left(a_{1}^{m}\right)}{\sqrt{\Re\left(a_{1}^{m}\right)^{2}+\Im\left(a_{1}^{m}\right)^{2}}} & \frac{\Im\left(a_{1}^{m}\right)}{\sqrt{\Re\left(a_{1}^{m}\right)^{2}+\Im\left(a_{1}^{m}\right)^{2}}} \\
\frac{-\Im\left(a_{1}^{m}\right)}{\left(\Re\left(a_{1}^{m}\right)^{2}+\Im\left(a_{1}^{m}\right)^{2}\right.} & \frac{\Re\left(a_{1}^{m}\right)}{\Re\left(a_{1}^{m}\right)^{2}+\Im\left(a_{1}^{m}\right)^{2}}
\end{array}\right] .
$$

The approximate covariance matrix $\Sigma_{a_{1}^{m}}^{\prime}$ for the magnitude and phase in radians is then given by [11]

$$
\Sigma_{a_{1}^{m}}^{\prime} \approx A \Sigma_{a_{1}^{m}} A^{T}=\sigma^{2}\left[\begin{array}{cc}
1 & 0 \\
0 & \left|a_{1}^{m}\right|^{-2}
\end{array}\right] .
$$

From (11), the variance of the magnitude is independent of signal amplitude, and the variance of the phase is inversely proportional to the magnitude squared of the signal being measured. It can be verified numerically or with Monte Carlo simulation that this is a good approximation when $\left|a_{1}^{m}\right| \gg \sigma$.

As $\left|a_{1}^{m}\right| \rightarrow 0$, the magnitude has a Rayleigh distribution, which results in more than a $50 \%$ decrease in variance of the magnitude, as shown in Fig. 2. The phase distribution becomes uniform with variance of $\pi^{2} / 3$ for phase in radians. Thus, (11) is not valid when the signal approaches the noise floor.

Although the approximation is only valid for large tone amplitudes, it is an intuitive figure for the instrument operator. When the amplitude is large, the variance of the phase decreases by approximately half for each $3-\mathrm{dB}$ increase in tone amplitude.

\section{B. Single NVNA Measurement}

The NVNA described in Section II removes the dependence of measured phase $\varphi\left(a_{1}^{m}\right)$ on that of the local oscillator by applying a nonlinear transformation (3). After the transformation, 
the complex values $\left(\Re\left(a_{1}\right)\right.$ and $\left.\Im\left(a_{1}\right)\right)$ of the transformed pseudowave $a_{1}$ are correlated and the variance depends on the amplitude of the signal and amplitude of the phase reference.

If the time-domain samples of the two receivers are corrupted by independent stationary narrowband additive Gaussian noise with equal variance, then the population covariance matrix for the raw measurements $\left(a_{1}^{m}\right.$ and $\left.R^{m}\right)$ is given by

$$
\Sigma=\left[\begin{array}{cccc}
\sigma^{2} & 0 & 0 & 0 \\
0 & \sigma^{2} & 0 & 0 \\
0 & 0 & \sigma^{2} & 0 \\
0 & 0 & 0 & \sigma^{2}
\end{array}\right]
$$

where $\sigma^{2}$ is the variance of $\Re\left(a_{1}^{m}\right), \Im\left(a_{1}^{m}\right), \Re\left(R^{m}\right)$, and $\Im\left(R^{m}\right)$.

The covariance matrix $\Sigma^{\prime}$ corresponding to the real and imaginary parts of the transformed pseudowave $\Re\left(a_{1}\right)$ and $\Im\left(a_{1}\right)$ is given by

$$
\begin{aligned}
\Sigma_{11}^{\prime} & =E\left[\Re\left(a_{1}\right)^{2}\right]-E\left[\Re\left(a_{1}\right)\right]^{2} \\
\Sigma_{22}^{\prime} & =E\left[\Im\left(a_{1}\right)^{2}\right]-E\left[\Im\left(a_{1}\right)\right]^{2} \\
\Sigma_{12}^{\prime} & =\Sigma_{21}^{\prime}=E\left[\Re\left(a_{1}\right) \Im\left(a_{1}\right)\right]-E\left[\Re\left(a_{1}\right)\right] E\left[\Im\left(a_{1}\right)\right]
\end{aligned}
$$

where $E \square$ is the expected value for the transformed pseudowave $a_{1}$.

The expected value for the transformed pseudowave $a_{1}$ is calculated by transforming the expected value for the raw measurements $a_{1}^{m}$ and $R^{m}$, where $a_{1}^{m}$ and $R^{m}$ have a multidimensional Gaussian distribution with variance $\Sigma$ [12]. The covariance matrix $\Sigma^{\prime}$ must then be evaluated by a multidimensional numerical integration. Therefore, Monte Carlo analysis is often the most practical method for obtaining accurate statistical information for this case, but this gives little insight into the system dynamics.

As for the single receiver, an approximation is a practical alternative to an exact formulation. Consider the linear approximation of (4) and (5)

$$
\left[\begin{array}{l}
\Re\left(a_{1}\right) \\
\Im\left(a_{1}\right)
\end{array}\right] \approx \mathrm{J}\left[\Re\left(a_{1}^{m}\right) \quad \Re\left(R^{m}\right) \quad \Im\left(a_{1}^{m}\right) \quad \Im\left(R^{m}\right)\right]^{T}
$$

where $\mathrm{J}$ is the Jacobian given by

$$
\mathrm{J}=\left[\begin{array}{llll}
\frac{\partial \Re\left(a_{1}\right)}{\partial \Re\left(a_{1}^{m}\right)} & \frac{\partial \Re\left(a_{1}\right)}{\partial \Re\left(R^{m}\right)} & \frac{\partial \Re\left(a_{1}\right)}{\partial \Im\left(a_{1}^{m}\right)} & \frac{\partial \Re\left(a_{1}\right)}{\partial \Im\left(R^{m}\right)} \\
\frac{\partial \Im\left(a_{1}\right)}{\partial \Re\left(a_{1}^{m}\right)} & \frac{\partial \Im\left(a_{1}\right)}{\partial \Re\left(R^{m}\right)} & \frac{\partial \Im\left(a_{1}\right)}{\partial \Im\left(a_{1}^{m}\right)} & \frac{\partial \Im\left(a_{1}\right)}{\partial \Im\left(R^{m}\right)}
\end{array}\right] .
$$

The covariance matrix $\Sigma^{\prime}$ for $\Re\left(a_{1}\right)$ and $\Im\left(a_{1}\right)$ is approximately given by

$$
\Sigma^{\prime} \approx \mathbf{J} \Sigma \mathbf{J}^{T}=\sigma^{2}\left[\begin{array}{ll}
w_{11} & w_{12} \\
w_{21} & w_{22}
\end{array}\right]
$$

where $w_{11}, w_{12}, w_{21}$, and $w_{22}$ are given in terms of the transformed pseudowave $a_{1}$ and transformed reference $R$ by

$$
\begin{aligned}
& w_{11}=1+\frac{\Im\left(a_{1}\right)^{2}}{|R|^{2}}=1+\frac{\left|a_{1}\right|^{2}}{|R|^{2}} \sin ^{2}(\phi) \\
& w_{22}=1+\frac{\Re\left(a_{1}\right)^{2}}{|R|^{2}}=1+\frac{\left|a_{1}\right|^{2}}{|R|^{2}} \cos ^{2}(\phi)
\end{aligned}
$$

$$
w_{12}=w_{21}=-\frac{\Re\left(a_{1}\right) \Im\left(a_{1}\right)}{|R|^{2}}=-\frac{\left|a_{1}\right|^{2}}{|R|^{2}} \cos (\phi) \sin (\phi)
$$

where $\phi=\varphi\left(a_{1}\right)$ is the phase of the transformed forward pseudowave $a_{1}, R=\left|R^{m}\right|$ is the transformed reference channel. ${ }^{4}$

The covariance matrix given by (18) will be called Covariance Model 1. This model assumes the receivers operate as described in Section III-A and the measurements are independent with equal variance. These assumptions may not be strictly true in a practical measurement setup. For the case where the measurements do not have equal variance, a new Covariance Model, called Covariance Model 2, is proposed in Section IV.

From Covariance Model 1, it is evident that the ratio $\left|a_{1}\right|^{2} /|R|^{2}$ should be small to minimize the variance of the corrected measurement. Generally the user has little control over the signal to be measured. However, the user can change the magnitude of the phase reference tones by changing the pulse repetition rate or attenuation. In addition, a filter could be used to reduce the crest factor of the phase reference waveform.

Often data is presented as magnitude and phase. The variance of the transformed magnitude $\left|a_{1}\right|$ is the same as the variance of the raw magnitude $\left|a_{1}^{m}\right|$ given by (8). The variance of the phase $\varphi\left(a_{1}\right)$ can be calculated from a numerical evaluation of the expected value, where the probability density function for the transformed phase is given by a convolution involving the probability density functions for the signal phase $\varphi\left(a_{1}^{m}\right)$ and reference phase $\varphi\left(R^{m}\right)$ [13].

With knowledge of the covariance matrix $\Sigma$ for the raw measurement, the approximate variance of the magnitude and phase can be calculated. The linearized transfer function for the magnitude and phase in terms of the raw measurements, derived by substituting (4) and (5) into the magnitude and phase equations, is

$$
\begin{aligned}
{\left[\begin{array}{c}
\left|a_{1}\right| \\
\varphi\left(a_{1}\right)
\end{array}\right] } & =\left[\begin{array}{c}
\sqrt{\Re\left(a_{1}^{m}\right)^{2}+\Im\left(a_{1}^{m}\right)^{2}} \\
\tan ^{-1}\left(\frac{\Im\left(a_{1}^{m}\right) \Re\left(R^{m}\right)-\Re\left(a_{1}^{m}\right) \Im\left(R^{m}\right)}{\Re\left(a_{1}^{m}\right) \Re\left(R^{m}\right)+\Im\left(a_{1}^{m}\right) \Im\left(R^{m}\right)}\right)
\end{array}\right] \\
& \approx \mathrm{B}\left[\Re\left(a_{1}^{m}\right) \Re\left(R^{m}\right) \Im\left(a_{1}^{m}\right) \Im\left(R^{m}\right)\right]^{T}
\end{aligned}
$$

where $\mathrm{B}$ is given by

$$
\mathrm{B}=\left[\begin{array}{cccc}
\frac{\Re\left(a_{1}^{m}\right)}{\left|a_{1}\right|} & 0 & \frac{\Im\left(a_{1}^{m}\right)}{\left|a_{1}\right|} & 0 \\
\frac{-\Im\left(a_{1}^{m}\right)}{\left|a_{1}\right|^{2}} & \frac{\Im\left(R^{m}\right)}{|R|^{2}} & \frac{\Re\left(a_{1}^{m}\right)}{\left|a_{1}\right|^{2}} & \frac{-\Re\left(R^{m}\right)}{|R|^{2}}
\end{array}\right] .
$$

The approximate covariance matrix $\Sigma_{a_{1}}^{\prime}$ for the magnitude $\left|a_{1}\right|$ and phase $\varphi\left(a_{1}\right)$ is then given by

$$
\Sigma_{a_{1}}^{\prime} \approx \mathrm{B} \Sigma \mathrm{B}^{T}=\sigma^{2}\left[\begin{array}{ll}
1 & 1 \\
0 & \frac{1}{\left|a_{1}\right|^{2}}+\frac{1}{|R|^{2}}
\end{array}\right] .
$$

From this approximation, the variance of the magnitude is independent of signal amplitude, while the variance of the phase is inversely proportional to the magnitude squared of the signal being measured and the amplitude squared of the signal in the reference receiver.

\footnotetext{
${ }^{4}$ Although by definition $R$ is real and nonnegative, the magnitude symbol is retained in the equations as a reminder.
} 
The magnitude distribution becomes Rayleigh as $\left|a_{1}\right| \rightarrow 0$ and the phase distribution becomes uniform. In this limit, (25) is no longer valid.

Similar results can be derived for the other transformed pseudowaves $b_{1}, a_{2}$, and $b_{2}$.

\section{Multiple NVNA Measurements}

It has been shown in Section III-B that the variance of the phase for the NVNA measurements are dependent on the amplitude of the measurement and the amplitude of the signal in the reference receiver. Typically, all four NVNA measurements are used. Since there is only one phase reference receiver for the system, there can be a high correlation of uncertainties across the different NVNA measurements.

The approximate variance of the phase for a single NVNA measurement channel can be extended to multiple measurement channels. Using the method outlined in Section III-B, the approximate covariance matrix for the phase measurements $\varphi\left(a_{1}\right)$, $\varphi\left(b_{1}\right), \varphi\left(a_{2}\right)$, and $\varphi\left(b_{2}\right)$ is given by

$\Sigma_{\phi}$

$$
\left[\begin{array}{cccc}
\approx \sigma^{2} & & & \\
\frac{\left|a_{1}\right|^{2}+|R|^{2}}{\left|a_{1}\right|^{2}|R|^{2}} & |R|^{-2} & |R|^{-2} & |R|^{-2} \\
|R|^{-2} & \frac{\left|b_{1}\right|^{2}+|R|^{2}}{\left|b_{1}\right|^{2}|R|^{2}} & |R|^{-2} & |R|^{-2} \\
|R|^{-2} & |R|^{-2} & \frac{\left|a_{2}\right|^{2}+|R|^{2}}{\left|a_{2}\right|^{2}|R|^{2}} & |R|^{-2} \\
|R|^{-2} & |R|^{-2} & |R|^{-2} & \frac{\left|b_{2}\right|^{2}+|R|^{2}}{\left|b_{2}\right|^{2}|R|^{2}}
\end{array}\right] .
$$

Therefore, to minimize the correlation between the transformed pseudowaves, the amplitude of the phase reference tones should be maximized.

\section{EMPIRICAL COVARIANCE MATRIX}

Section III has detailed the theoretical derivation of the covariance matrix for mixer-based NVNA measurements. This derivation made the assumption that the signal and phase reference measurements were corrupted by independent stationary narrowband additive Gaussian noise with equal variance. In a practical measurement system, this assumption may not be valid, which requires that the model be modified to account for alternative noise sources.

To verify the theoretical derivation for the approximate covariance matrix for a single NVNA measurement from Section III-B, measurements were taken with the measurement system described in Section II. It was found that the approximate covariance matrix coefficients (19)-(21) were not appropriate for all measurement scenarios.

To account for this observation, a new model was empirically derived by varying the signal amplitude and reference tone amplitude. It was observed that the variance of the measurements with narrow IF bandwidth (small $\sigma^{2}$ ) was a strong function of signal magnitude $\left|a_{1}\right|$ independent of the phase reference magnitude $|R|$. To account for this observation, an additional factor $F_{n}$ that depends on the noise from the phase reference was

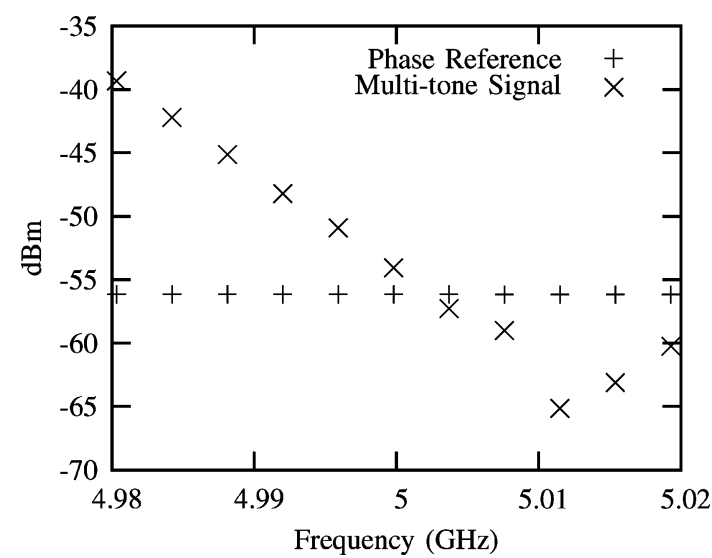

Fig. 3. Measured magnitude spectrum of a multitone signal and magnitude spectrum of the phase reference. The phase reference clock frequency was set to $3.9 \mathrm{MHz}$.

added to the covariance models. The new magnitude/phase covariance matrix (25) is given by

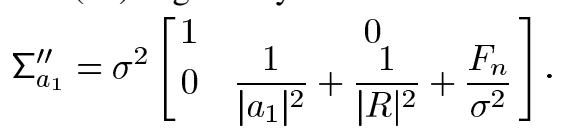

A new model for the variance of the real and imaginary parts of $a_{1}$ can then be derived by transforming the covariance matrix for magnitude/phase (27) to the covariance matrix for the real/imaginary values using the linearization method. The new model, i.e., Covariance Model 2, is proposed as

$$
\begin{aligned}
& \Sigma_{11}^{\prime} \approx \sigma^{2} w_{11}+F_{n}\left|a_{1}\right|^{2} \sin ^{2}(\phi) \\
& \Sigma_{22}^{\prime} \approx \sigma^{2} w_{22}+F_{n}\left|a_{1}\right|^{2} \cos ^{2}(\phi) \\
& \Sigma_{12}^{\prime}=\Sigma_{21}^{\prime} \approx \sigma^{2} w_{12}-F_{n}\left|a_{1}\right|^{2} \cos (\phi) \sin (\phi)
\end{aligned}
$$

where $w_{11}, w_{12}$, and $w_{22}$ are from Covariance Model 1 (19)-(21) and $F_{n}$ is a factor that depends on the noise from the phase reference.

The covariance matrix elements $\Sigma_{11}^{\prime}$ and $\Sigma_{22}^{\prime}$ are the variance of the real and imaginary parts of $a_{1}$, respectively. The covariance matrix element $\Sigma_{12}^{\prime}=\Sigma_{21}^{\prime}$ is the covariance, which is dependent on the direction and how much the real and imaginary parts vary together in the complex domain.

Section V compares Covariance Model 2 to measurements made with the system described in Section II.

\section{MEAsurements}

Some measurement examples to verify Covariance Model 2 are given here. The measurements were selected to evaluate the model with different signal amplitudes, phase reference amplitudes, and IF bandwidth conditions. Several multitone signals consisting of 11 pseudorandom phase tones spaced $3.9 \mathrm{MHz}$ apart centered around $4.9998 \mathrm{GHz}(4.9998-\mathrm{GHz}$ carrier with five tones spaced on either side) were generated with an $\mathrm{Ag}$ ilent E8267C PSG. The tone amplitudes of the multitone signals were chosen to span a range of $26 \mathrm{~dB}$ (limited by the generator). To cover a wider dynamic range $(>26 \mathrm{~dB})$, multiple measurements were performed where the carrier amplitude was adjusted. The results of those multiple measurements with different carrier amplitudes were combined and the combined results are plotted here. An example of one of the multitone signals is shown in Fig. 3. The phase reference repetition rate was set 


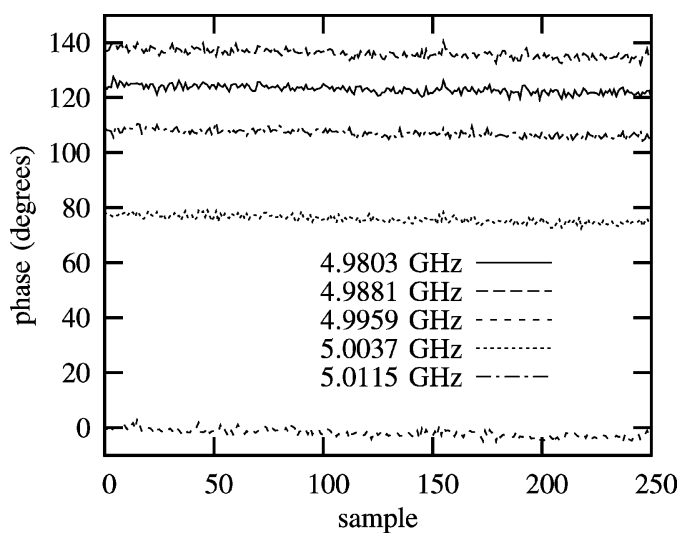

Fig. 4. Phase measurement repeated over a period of time for five tones (limited number of tones plotted to make the graph easier to view) of the multitone signal from Fig. 3. Although the sources and measurement system are phase locked to a common reference, the measured phase varies slowly over time due to drift and imperfect phase locking. For these measurements, the phase varies in a range of approximately $5^{\circ}$ over the measurement time period (less than $1 \mathrm{~h}$ ).

to $3.9 \mathrm{MHz}$ for most experiments, unless stated otherwise. The phase reference was driven with a low phase-noise microwave signal generator (Agilent E4437B) and the output frequency was reduced to $3.9 \mathrm{MHz}$ with a frequency divider to further reduce the phase noise. A trial of 250 measurements was performed to calculate the sample covariance matrix corresponding to each tone in the multitone signal. The signal source (Agilent E8267C), phase reference signal generator, and the receiver hardware were phase locked via the $10-\mathrm{MHz}$ reference, hence, the phase variation with time is slow, as seen in Fig. 4. For this plot, the phase varies in a range of approximately $5^{\circ}$ over the measurement time period (less than $1 \mathrm{~h}$ ). The variation has a random component and deterministic component.

To remove the deterministic component from the measured phase, a linear regression was performed on the phase and subtracted from the measured phase such that the resulting phases have a mean around 0 . After the deterministic component was removed, the magnitude and phase were converted to rectangular coordinates and the sample covariance matrix $(\mathrm{V})$ with elements $\mathrm{V}_{11}, \mathrm{~V}_{12}, \mathrm{~V}_{21}$, and $\mathrm{V}_{22}$ was calculated for $a_{1}$.

The resulting sample points in rectangular coordinates, after removing the deterministic component, for a few power levels and $100-\mathrm{Hz}$ resolution bandwidth are shown in Fig. 5. For low power levels, the width of the oval defining the $95 \%$ confidence region for the mean remains fairly constant, while the height increases with increasing signal amplitude.

The sample covariance matrix was calculated using the following unbiased estimators:

$$
\begin{aligned}
& \mathrm{V}_{11}=\frac{1}{N-1} \sum_{i=0}^{N-1}\left[\mathbf{x}_{i}-\overline{\mathbf{x}}\right]^{2} \\
& \mathrm{~V}_{22}=\frac{1}{N-1} \sum_{i=0}^{N-1}\left[\mathbf{y}_{i}-\overline{\mathbf{y}}\right]^{2} \\
& \mathrm{~V}_{12}=\mathrm{V}_{21}=\frac{1}{N-1} \sum_{i=0}^{N-1}\left[\mathbf{x}_{i}-\overline{\mathbf{x}}\right]\left[\mathbf{y}_{i}-\overline{\mathbf{y}}\right]
\end{aligned}
$$

where $N=250$ is the number of samples, $\mathbf{x}$ is a vector of samples from $\Re\left(a_{1}\right), \mathbf{y}$ is a vector of samples from $\Im\left(a_{1}\right), \overline{\mathbf{x}}$ is the mean of the vector $\mathbf{x}$, and $\overline{\mathbf{y}}$ is the mean of the vector $\mathbf{y}$.

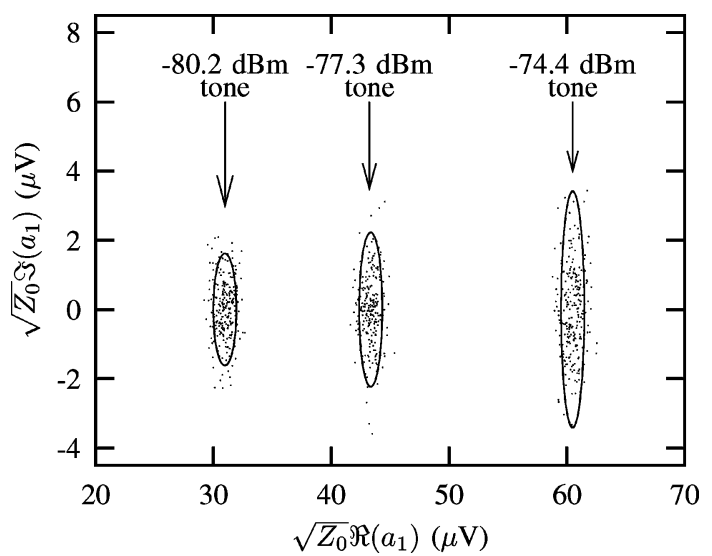

Fig. 5. Plot of the samples used to calculate the variance of the measured $-80.2-,-77.3-$, and -74.4-dBm tones. The measurements used for this plot are from a lower amplitude multitone signal than that plotted in Fig. 3. A 95\% confidence region for the mean of each tone is superimposed on the sample points (see [14]). The covariance matrix element $V_{11}$ is proportional to the width and $V_{22}$ is proportional to the height of the oval defining the confidence region. For low power levels, the width $\left(\mathrm{V}_{11}\right)$ remains fairly constant, while the height $\left(\mathrm{V}_{22}\right)$ increases with increasing signal amplitude.

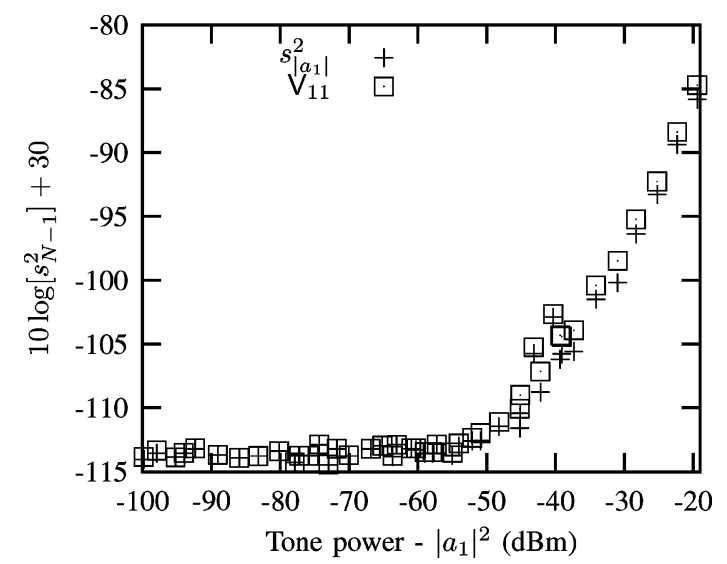

Fig. 6. Plot of the sample variance of the magnitude $s_{\left|a_{1}\right|}^{2}$ and the sample variance of the real part $V_{11}$ when the phase reference is driven by the Agilent E4437 signal generator. The variance of the magnitude is constant at low tone powers and then increases with increasing tone power above $-55 \mathrm{dBm}$. This suggests that the magnitude $\left|a_{1}\right|$ does not have a Rice distribution. The sample variance of the real part $V_{11}$ is highly correlated with the sample variance of the magnitude $s_{\left|a_{1}\right|}^{2}$.

Where appropriate, the sample variance $s_{N-1}^{2}$ is plotted using the transformation $10 \log \left[s_{N-1}^{2}\right]+30$, which is a useful transformation for evaluating noise power.

The discussion will proceed with analysis of the variance of the magnitude and the variance of the phase. The sample variance of the real $\left(\mathrm{V}_{11}\right)$ and imaginary $\left(\mathrm{V}_{22}\right)$ parts of $a_{1}$ will then be compared to the variance predicted by Covariance Model 2 .

\section{A. Variance of the Magnitude}

The sample variance of the magnitude $s_{\left|a_{1}\right|}^{2}$ is plotted in Fig. 6. If the time-domain samples of $a_{1}^{m}$ are corrupted by independent stationary narrowband additive Gaussian noise, then the magnitude $\left|a_{1}\right|$ would have a Rice distribution with variance given by (8). From Fig. 2, the variance of a random variable with a Rice distribution is fairly constant when the tone amplitude is much greater than the standard deviation. 


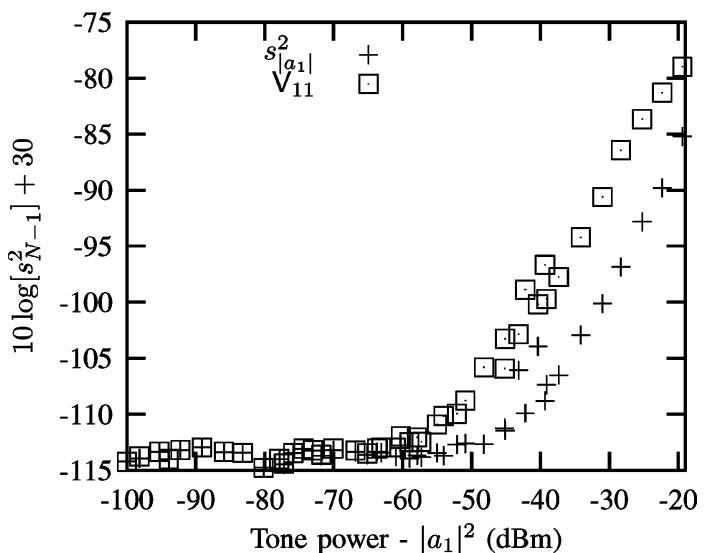

Fig. 7. Plot of the sample variance of the magnitude $s_{\left|a_{1}\right|}^{2}$ and the sample variance of the real part $\mathrm{V}_{11}$ when the phase reference is driven by the Agilent 83620 signal generator. The sample variance of the real part $V_{11}$ does not correlate with the sample variance of the magnitude $s_{\left|a_{1}\right|}^{2}$ above $-60 \mathrm{dBm}$.

However, the variance starts to increase above $-55 \mathrm{~dB}$ for the measurements considered. Therefore, the magnitude $\left|a_{1}\right|$ does not have a Rice distribution and the assumption that the samples are corrupted by independent stationary narrowband additive Gaussian noise is invalid.

The phase noise of the signal generator used to drive the phase reference can effect the variance of some measurement quantities. The sample variance of the magnitude $s_{\left|a_{1}\right|}^{2}$, using an Agilent 83620 signal generator instead of the Agilent E4437, is plotted in Fig. 7. The Agilent 83620 has moderate phase noise compared with the Agilent E4437 signal generator. Comparing Figs. 6 and 7, it can be seen that the sample variance of the magnitude $s_{\left|a_{1}\right|}^{2}$ is not affected by the signal generator used to drive the phase reference.

A model could easily be fitted to the variance of the magnitude for this particular measurement setup. However, additional work is required to understand the nature of this uncertainty such that a generic model can be derived for the measurement system.

\section{B. Variance of the Phase}

A comparison of the sample variance of $a_{1}$ phase when the phase reference is driven by the Agilent E4437 and 83620 signal generators is presented in Fig. 8. The variance observed at high tone power with either generator is greater than what would be expected if the measurements of $a_{1}^{m}$ were corrupted by stationary narrowband additive Gaussian noise. Using different signal generators with different phase-noise specifications to drive the phase reference gives an indication that the noise source might be linked to the phase noise of the signal generator driving the phase reference.

The Agilent 83620 has higher phase noise, which is believed to result in the higher variance observed when using this signal generator. The $F_{n}$ factor was proposed in Section IV as an empirical factor to account for the noise produced by the phase reference. A model of the variance (27), which includes the $F_{n}$ factor, is superimposed on the plot for the measurement using the Agilent E4437 signal generator. This model can then be

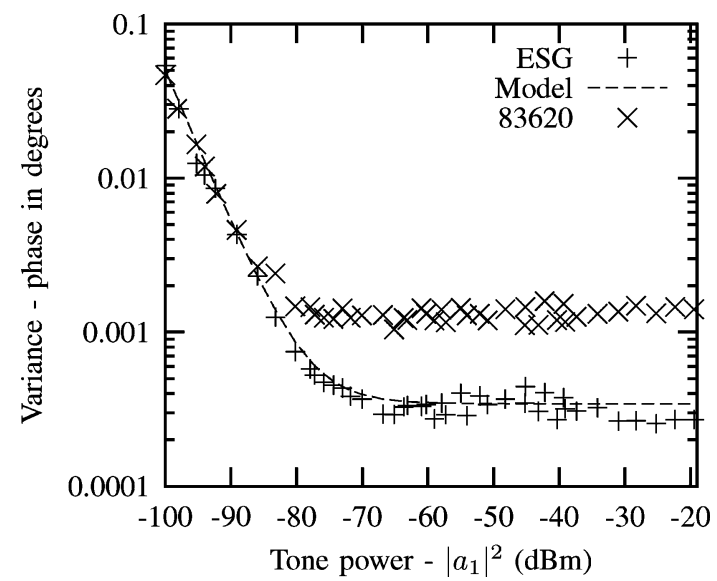

Fig. 8. Comparison of $a_{1}$ phase variance when the phase reference is driven by the Agilent E4437 and 83620 signal generators. A model of the variance (27), which includes the $F_{n}$ factor, is superimposed on the plot for the measurement using the Agilent E4437 signal generator.

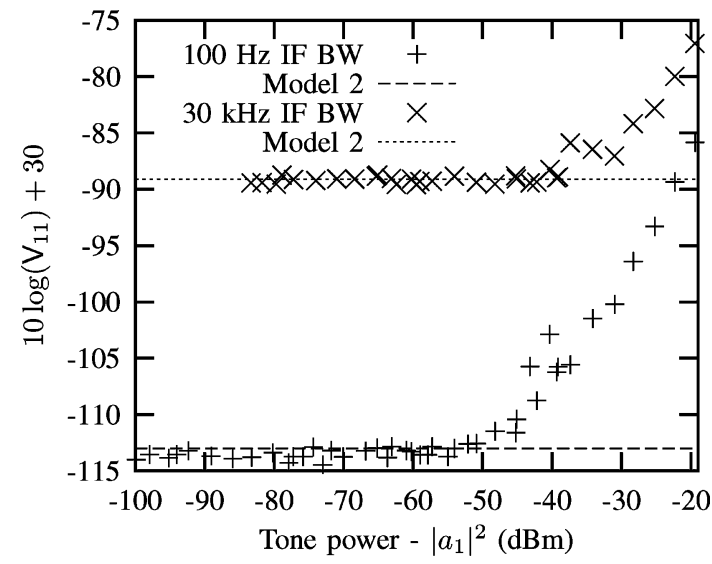

Fig. 9. Comparison of the sample variance $\mathrm{V}_{11}$ and the variance predicted by Covariance Model 2. The instrument IF bandwidth was set to $100 \mathrm{~Hz}$ and $30 \mathrm{kHz}$. A low phase-noise signal generator (Agilent E4437) was used to drive the phase reference. The model is able to predict the variance up to $-55 \mathrm{dBm}$.

used, for example, to weight the phase detrending cost function [15].

\section{Analysis of $\mathrm{V}_{11}$}

A comparison of the sample variance $V_{11}$ and the variance predicted by Covariance Model 2 for wide IF bandwidth $(30 \mathrm{kHz})$ and narrow IF bandwidth $(100 \mathrm{~Hz})$ is shown in Fig. 9. The model correctly predicts the variance for tone powers up to -40 and $-55 \mathrm{dBm}$ for wide IF bandwidth $(30 \mathrm{kHz})$ and narrow IF bandwidth $(100 \mathrm{~Hz})$, respectively. Above these tone powers, the variance increases above that predicted by the model.

The sample variance of the real part $\bigvee_{11}$ is compared to the variance of the magnitude $s_{\left|a_{1}\right|}^{2}$ in Figs. 6 and 7 when the phase reference is driven by the Agilent E4437 and 86320 signal generators, respectively. When the phase reference is driven by the Agilent E4437, the sample variance of the real part $V_{11}$ is highly correlated with the sample variance of the magnitude $s_{\left|a_{1}\right|}^{2}$. However, when the phase reference is driven by the 86320 , the sample variance of the real part $V_{11}$ does not follow the sample variance of the magnitude $s_{\left|a_{1}\right|}^{2}$. 


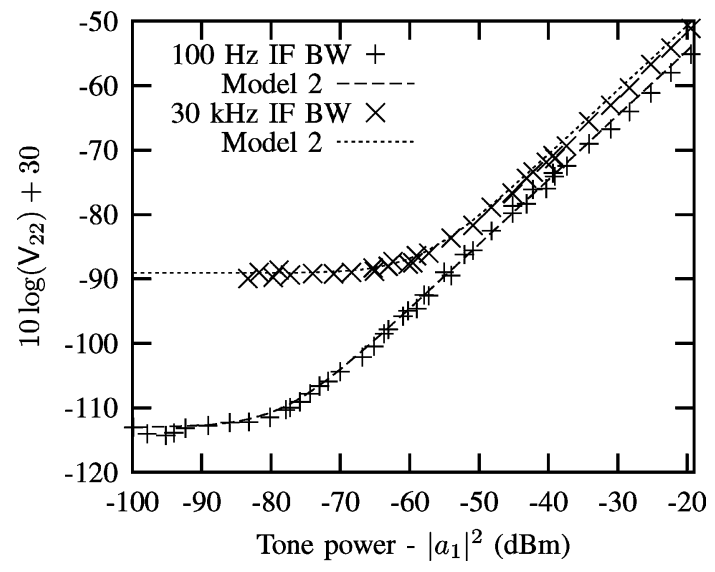

Fig. 10. Comparison of the sample variance $\mathrm{V}_{22}$ and the variance predicted by Covariance Model 2. The instrument IF bandwidth was set to $100 \mathrm{~Hz}$ and $30 \mathrm{kHz}$. A low phase-noise signal generator (Agilent E4437) was used to drive the phase reference. For these measurements, the model is able to predict the variance for both wide $(30 \mathrm{kHz})$ and narrow $(100 \mathrm{~Hz}) \mathrm{IF}$ bandwidths.

This suggests that the sample variance of the real part $\mathrm{V}_{11}$ has a relationship with the variance of the magnitude and the phase noise of the phase reference. Additional work is, therefore, required to understand the system noise sources and interaction of those sources on the observed variance.

\section{Analysis of $\mathrm{V}_{22}$}

A comparison of the sample variance of the imaginary part $\left(\mathrm{V}_{22}\right)$ and the variance predicted by Covariance Model 2 for different IF bandwidths is shown in Fig. 10. For these measurements, the model is able to predict the variance for both wide $(30 \mathrm{kHz})$ and narrow $(100 \mathrm{~Hz}) \mathrm{IF}$ bandwidths across the entire measurement range.

The applicability of Covariance Model 2 over the entire measurement range is attributed to the inclusion of the additional factor $F_{n}$. The factor $F_{n}$ accounts for the noise from the phase reference, which, at higher tone powers, appears to be the dominant noise source contributing to $V_{22}$ for these measurements.

A comparison of the sample variance $V_{22}$ and the variance predicted by Covariance Model 2 for different phase reference clock frequencies is shown in Fig. 11. The phase reference used in the prototype system generates a single impulse every clock cycle. Hence, altering the phase reference clock frequency is an effective method for varying the reference tone amplitude $|R|$. This is because there is an approximately linear relationship between clock frequency and reference tone amplitude $|R|$. The model is able to predict the change in variance for different phase reference clock frequencies (different phase reference tone amplitudes $|R|$ ).

\section{E. Analysis of Covariance Model 2}

The empirically derived model, i.e., Covariance Model 2, was able to predict $V_{11}$ for tone powers up to $-55 \mathrm{dBm}$ and $V_{22}$ for tone powers up to $-20 \mathrm{dBm}$, various phase reference tone amplitudes $|R|$ (phase reference clock frequencies), and various IF bandwidths. Additional work is required to improved current understanding of the system noise sources to enable the model of $V_{11}$ to predict variance over a wider range of tone amplitudes.

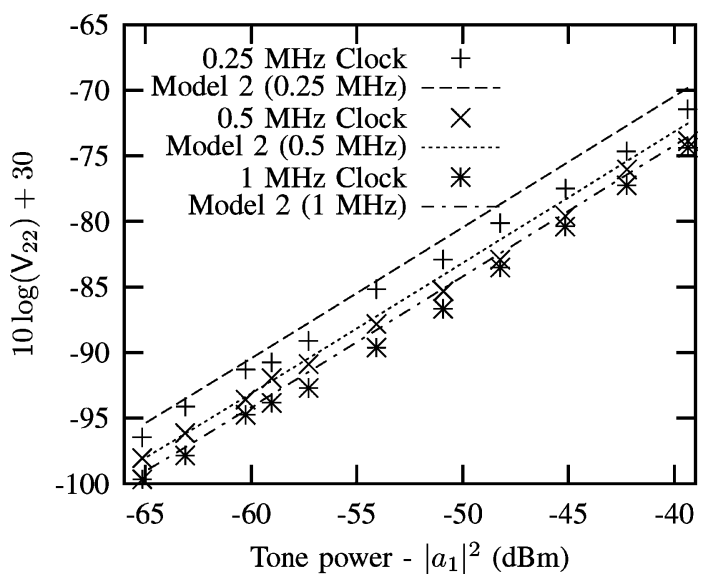

Fig. 11. Comparison of the sample variance $V_{22}$ and the variance predicted by Covariance Model 2 for different phase reference clock frequencies. The instrument IF bandwidth was set to $100 \mathrm{~Hz}$. The Agilent E4437 signal generator was used to drive the phase reference. For these measurements, the model is able to predict the change in variance for different phase reference clock frequencies (change in phase reference magnitude $|R|$ ). Note that the clock frequency reported on the plot is approximate for clarity. $0.25-\mathrm{MHz}$ clock is $0.24375 \mathrm{MHz}$, $0.5-\mathrm{MHz}$ clock is $0.4875 \mathrm{MHz}$, and $1-\mathrm{MHz}$ clock is $0.975 \mathrm{MHz}$.

Covariance Model 2 can be used to obtain the approximate covariance matrix for $a_{1}$ given $a_{1}$ and the magnitude of the phase reference $|R|$. The covariance matrix can then be used for estimating the uncertainty of measurements and for fitting models.

The approximate covariance matrix also gives the user an intuitive understanding of system behavior. From (28)-(30), it is apparent that the variance can be minimized by maximizing the amplitude of the phase reference tones while minimizing phase noise from the phase reference.

The phase reference used for the prototype is a repetitive impulse in the time domain. The impulse contains its energy in a narrow segment of time compared to the clock period. The amplitude of the impulse in the time domain is limited by the reference receiver. To increase the amplitude of the tones in the frequency domain, the impulse repetition rate can be increased, an all-pass filter can be used to disperse the energy over a wider segment in the time domain, or a tunable filter can be used to reduce the undesired energy incident on the receiver.

Phase noise from the phase reference can be minimized by driving the phase reference with a low phase-noise signal generator and using dividers on the output. The effectiveness of dividers for reducing phase noise is set by the noise contribution of the dividers.

\section{CONCLUSION}

An approximate covariance matrix has been derived for measurements made with a mixer-based NVNA. The covariance matrix is required to weight the cost function when fitting models, weight the signal alignment problem, and for calculating uncertainty bounds. The approximation allows straightforward estimation of the covariance matrix from limited information about the measurements performed. The approximation has been verified by measurements up to $-55-\mathrm{dBm}$ tone power incident on the receiver, various IF bandwidths, and phase reference tone amplitudes. 


\section{REFERENCES}

[1] P. S. Blockley, D. Gunyan, and J. B. Scott, "Mixer-based, vector-corrected, vector signal/network analyzer offering $300 \mathrm{kHz}-20 \mathrm{GHz}$," in IEEE MTT-S Int. Microw. Symp. Dig., Jun. 2005, pp. 1497-1500.

[2] D. F. Williams, P. D. Hale, T. S. Clement, and J. M. Morgan, "Calibrating electro-optic sampling systems," in IEEE MTT-S Int. Microw. Symp. Dig., 2001, vol. 3, pp. 1527-1530.

[3] R. B. Marks and D. F. Williams, "A general waveguide circuit theory," J. Res. NIST, vol. 97, no. 5, pp. 533-562, Sep.-Oct. 1992.

[4] P. S. Blockley, J. B. Scott, D. Gunyan, and A. E. Parker, "Noise considerations when determining phase of large-signal microwave measurements," IEEE Trans. Microw. Theory Tech., vol. 54, no. 8, pp. 3182-3190, Aug. 2006

[5] S. Rice, "Statistical properties of a sine-wave plus random noise," Bell Syst. Tech. J., vol. 27, pp. 109-157, Jan. 1948.

[6] S. Rice, "Mathematical analysis of random noise," Bell Syst. Tech. J., vol. 23, pp. 282-332, Jul. 1944.

[7] S. Rice, "Mathematical analysis of random noise," Bell Syst. Tech. J., vol. 24, pp. 46-156, Jan. 1945.

[8] J. E. Dennis, D. M. Gay, and R. E. Welsch, "Algorithm 573: NL2SOL-An adaptive nonlinear least-squares algorithm," ACM Trans. Math. Softw., vol. 7, no. 3, pp. 369-383, Sep. 1981.

[9] P. T. Boggs and J. E. Rogers, Contemporary Mathematics. Providence, RI: Amer. Math. Soc., 1990, vol. 112, pp. 183-194.

[10] J. R. Donaldson and R. B. Schnabel, "Computational experience with confidence intervals for nonlinear least squares," Technometrics, vol. 29, no. 1, pp. 67-82, Feb. 1987.

[11] S. J. Press, Applied Multivariate Analysis. New York: Holt, Rinehart and Winston, 1972.

[12] M. C. Jeruchim, Simulation of Communication Systems: Modeling, Methodology, and Techniques. New York: Kluwer, 2000.

[13] A. Papoulis, Probability, Random Variables, and Stochastic Processes. New York: McGraw-Hill, 1965.

[14] B. D. Hall, "Calculating measurement uncertainty for complex-valued quantities," Meas. Sci. Technol., vol. 14, no. 3, pp. 368-375, Feb. 2003.

[15] K. A. Remley, D. F. Williams, D. M. M.-P. Schreurs, G. Loglio, and A Cidronali, "Phase detrending for measured multisine signals," in 61st ARFTG Conf. Dig., Jun. 2003, pp. 73-83.

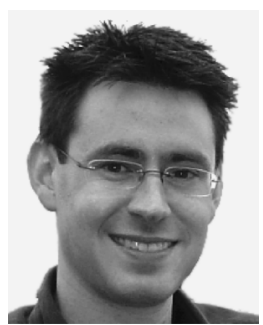

Peter Stuart Blockley (S'03-M'07) received the B.Sc. and Ph.D. degrees from Macquarie University, Sydney, N.S.W., Australia, in 2002 and 2007, respectively.

$\mathrm{He}$ is currently with South Pacific IT, Sydney, N.S.W., Australia. He has authored or coauthored six publications.

Dr. Blockley was the recipient of the 1997 Merit Award in Electronics Technology, the 2005 Automatic RF Techniques Group (ARFTG) Best Interactive Session Paper Award, and the 2005 Macquarie University Innovation Award for research in partnership with Agilent Technologies.

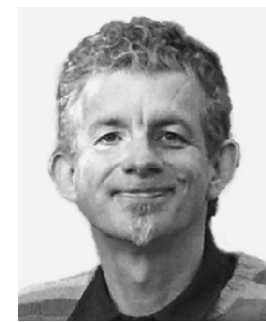

Jonathan Brereton Scott (M'80-SM'99) became the Foundation Professor in Electronic Engineering with the University of Waikato, Hamilton, New Zealand, in 2006. From 1998 to 2006, he was with the Microwave Technology Center, Hewlett-Packard (now Agilent Technologies), Santa Rosa, CA, where he was responsible for advanced measurement systems. In 1997 and 1998, he was Chief Engineer with RF Technology, Sydney, N.S.W., Australia. Prior to 1997, he was with the Department of Electrical Engineering, University of Sydney. He is a Professorial Fellow of Macquarie University. He has served on committees of the Standards Association of Australia and the NRC Review Panel for the National Institute of Standards and Technology (NIST). He has authored numerous refereed publications, several book chapters, and a textbook. $\mathrm{He}$ holds number of patents.

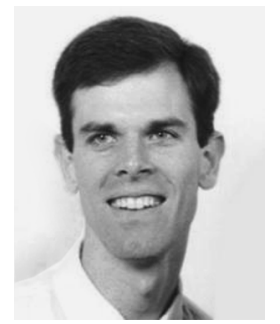

Daniel Gunyan (M'97-SM'07) received the B.S degree in electrical and computer engineering and M.S. degree in electrical engineering from Brigham Young University, Provo, UT, in 1996 and 1997, respectively.

Since 1997, he has been with Agilent Technologies (formerly Hewlett-Packard), Santa Rosa, CA, where he is involved in both manufacturing and design of microwave and millimeter-wave components for measurement systems and radio transceivers. $\mathrm{He}$ currently develops test systems and measuremen methods for nonlinear characterization and modeling of high-frequency devices, components, and systems.

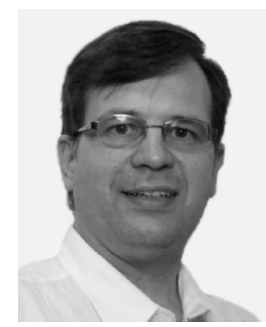

Anthony Edward Parker (S'84-M'90-SM'95) received the B.Sc., B.E., and Ph.D. degrees from The University of Sydney, Sydney, Australia, in 1983, 1985, and 1992, respectively.

$\mathrm{He}$ is currently a Professor with Macquarie University, Sydney, N.S.W., Australia, where he is also Director of the Centre for Microwave and Wireless Applications. His research has produced accurate circuit simulation techniques such as used in field-effect transistor (FET) and HEMT models. He has authored or coauthored over 140 publications. His recent research has been in the area of intermodulation in broadband circuits and systems, including a major project with Agilent Technologies Inc. and Mimix Broadband Inc.

Prof. Parker is a member of the Institution of Engineers, Australia and the National Committee for Radio Science. He is a committee member of the IEEE Antennas and Propagation (AP)/Microwave Theory and Techniques (MTT) N.S.W Local Chapter. 